\title{
MR Imaging in Menière Disease: Is the Contact between the Vestibular Endolymphatic Space and the Oval Window a Reliable Biomarker?
}

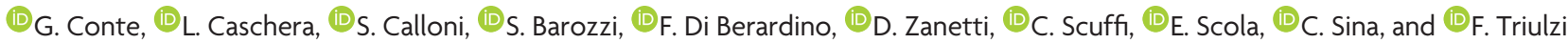

\begin{abstract}
BACKGROUND AND PURPOSE: No reliable MR imaging marker for the diagnosis of Menière disease has been reported. Our aim was to investigate whether the obliteration of the inferior portion of the vestibule and the contact with the stapes footplate by the vestibular endolymphatic space are reliable MR imaging markers in the diagnosis of Menière disease.
\end{abstract}

MATERIALS AND METHODS: We retrospectively enrolled 49 patients, 24 affected by unilateral sudden hearing loss and 25 affected by definite Menière disease, who had undergone a 4-hour delayed 3D-FLAIR sequence. Two readers analyzed the MR images investigating whether the vestibular endolymphatic space bulged in the third inferior portion of the vestibule contacting the stapes footplate. This sign was defined as the vestibular endolymphatic space contacting the oval window.

RESULTS: We analyzed 98 ears: 27 affected by Menière disease, 24 affected by sudden sensorineural hearing loss, and 47 that were healthy. The vestibular endolymphatic space contacting the oval window showed an almost perfect interobserver agreement (Cohen $\kappa=$ $0.87 ; 95 \% \mathrm{Cl}, 0.69-1)$. The vestibular endolymphatic space contacting oval window showed the following: sensitivity $=81 \%$, specificity $=$ $96 \%$, positive predictive value $=88 \%$, and negative predictive value $=93 \%$ in differentiating Menière disease ears from other ears. The vestibular endolymphatic space contacting the oval window showed the following: sensitivity $=81 \%$, specificity $=96 \%$, positive predictive value $=96 \%$, negative predictive value $=82 \%$ in differentiating Menière disease ears from sudden sensorineural hearing loss ears.

CONCLUSIONS: The vestibular endolymphatic space contacting the oval window has high specificity and positive predictive value in differentiating Menière disease ears from other ears, thus resulting in a valid tool for ruling in Menière disease in patients with mimicking symptoms.

ABBREVIATIONS: AAO-HNS = American Academy of Otolaryngology-Head and Neck Surgery; $\mathrm{BLB}=$ blood-labyrinthine barrier; $\mathrm{CH}=\mathrm{cochlear}$ hydrops; $\mathrm{HC}=$ healthy control; $M D=$ Menière disease; $\mathrm{nMD}=$ normal in a patient with $\mathrm{MD} ; \mathrm{NPV}=$ negative predictive value; $\mathrm{PPV}=$ positive predictive value; $\mathrm{SSHL}=$ sudden sensorineural hearing loss; $\mathrm{VEH}=$ vestibular endolymphatic hydrops; $\mathrm{VES}=$ vestibular endolymphatic space; VESCO = vestibular endolymphatic space contacting the oval window

M eniére disease $(\mathrm{MD})$ is a relatively common disorder, with a prevalence of 200-500 per 100,000, characterized by fluctuating hearing loss, intermittent vertigo, tinnitus, and aural fullness. ${ }^{1}$ MD is characterized by the presence of vestibular endolymphatic hydrops (VEH), a distension of the vestibular endolymphatic space (VES) of the inner ear. However, VEH might

Received May 9, 2018; accepted after revision August 20.

From the Neuroradiology Unit (G.C., E.S., C. Sina, F.T.), Fondazione IRCCS

Ca'Granda Ospedale Maggiore Policlinico, Milan, Italy; Postgraduation School of Radiodiagnostics (L.C., S.C.) and Departments of Medicine and Surgery (C. Scuffi) and Pathophysiology and Transplantation (F.T.), Università degli Studi di Milano, Milan, Italy; and Audiology Unit (S.B., F.D.B., D.Z.), Department of Clinical Sciences and Community, Fondazione IRCCS Ca'Granda Ospedale Maggiore Policlinico, Università degli Studi di Milano, Milan, Italy.

Please address correspondence to Giorgio Conte, MD, Neuroradiology Unit, Fondazione IRCCS Ca'Granda Ospedale Maggiore Policlinico, Via Francesco Sforza 35, 20122 Milan, Italy; e-mail: giorgioconte.unimed@gmail.com; @GiorgioConte86

http://dx.doi.org/10.3174/ajnr.A5841 not represent the sole cause of the symptoms. Following the assumption of Merchant et al, "It seems that all patients with classical symptoms of Menière's disease have $\mathrm{VEH}$, but not vice versa, as not all patients with hydrops have Menière's disease symptoms." "A diagnosis of definite MD is made by the presence of $\geq 2$ episodes of vertigo, audiometrically documented low-to-medium frequency sensorineural hearing loss in 1 ear, or fluctuating aural fullness. $^{3}$

MR imaging has recently been used in the diagnosis of MD because the presence of VEH can be demonstrated in vivo with a 3D-FLAIR sequence 4 hours after the intravenous administration of a contrast agent. ${ }^{4}$ Data emerging from the literature about MR imaging of $\mathrm{VEH}$ in patients with MD are still discordant/uneven. Several diagnostic criteria have been proposed to evaluate the degree of the $\mathrm{VEH}$, the most relevant/common being those of Nakashima et $\mathrm{al}^{5}$ a quantitative method that assesses the VES, 
Table 1: Scanning parameters (3T)

\begin{tabular}{|c|c|c|c|c|}
\hline & $\begin{array}{c}\text { Pre- and } \\
\text { Postcontrast } \\
\text { TI FSE }\end{array}$ & $\begin{array}{l}\text { Pre- and } \\
\text { Postcontrast } \\
\text { 3D-FLAIR }\end{array}$ & $\begin{array}{c}\text { 3D } \\
\text { T2-SSFP }\end{array}$ & $\begin{array}{c}\text { Delayed } \\
\text { Postcontrast } \\
\text { 3D-FLAIR }\end{array}$ \\
\hline Plane & Axial & Axial & Axial & Axial \\
\hline TR (ms) & 500 & 6000 & 1500 & 7600 \\
\hline TE (ms) & 10 & 350 & 194 & 345 \\
\hline TI (ms) & / & 2350 & / & 2100 \\
\hline Fat saturation & SPIR & SPIR & / & SPAIR \\
\hline TSE factor & 3 & 182 & 40 & 100 \\
\hline Flip angle & $90^{\circ}$ & $90^{\circ}$ & $90^{\circ}$ & $90^{\circ}$ \\
\hline Slice thickness (mm) & 1.5 & 1 & 0.6 & 0.6 \\
\hline Slices (no.) & 15 & 30 & 22 & 40 \\
\hline $\mathrm{FOV}\left(\mathrm{mm}^{2}\right)$ & $180 \times 160$ & $230 \times 190$ & $140 \times 140$ & $200 \times 255$ \\
\hline Matrix & $256 \times 205$ & $232 \times 229$ & $264 \times 248$ & $250 \times 252$ \\
\hline Parallel imaging & No & Yes & No & Yes \\
\hline Averages & 4 & 6 & 1 & 4 \\
\hline Scan time & $2 \mathrm{~min} 51 \mathrm{sec}$ & $10 \min 6 \mathrm{sec}$ & $6 \mathrm{~min} 35 \mathrm{sec}$ & $9 \mathrm{~min} 15 \mathrm{sec}$ \\
\hline
\end{tabular}

Note:-SSFP indicates steady-state free precession; SPIR, spectral presaturation with inversion recovery; SPAIR, spectral attenuated inversion recovery; 3D FLAIR, Three dimensionally Fluid Attenuated Inversion Recovery; FSE, Fast spin echo; TR, time of repetition; TE, time of echo; TI, time of inversion; TSE, Turbo Spin-Echo; FOV, field of view; /, specific parameter is not available.

calculating the ratio of the area of the VES to the entire vestibule (VES/vestibule ratio) in the axial plane and defining VEH as present if this ratio is $>33 \%$. More recently, Attyé et $\mathrm{al}^{6}$ have established a morphology-based method: Saccular hydrops is considered when a saccule-to-utricle ratio of $\geq 1$ is present. However, both of these scores seem to lack high accuracy in differentiating MD from other otologic disorders. ${ }^{7}$

There is an unmet clinical need to understand the role of the contact between the VES and the stapes footplate. Our aim was to investigate whether the obliteration of the inferior portion of the vestibule with the contact between the ES and the stapes footplate is a reliable MR imaging marker in differentiating MD ears from healthy ears and from those affected by other otologic disorders. In addition, we assessed whether the blood-labyrinthine barrier (BLB) breakdown is associated with the presence of symptoms on the MR image.

\section{MATERIALS AND METHODS \\ Subject Enrollment}

This study was approved by the Fondazione IRCCS Ca' Granda Ospedale Maggiore Policlinico institutional review board; patient informed consent was waived. We retrospectively enrolled consecutive patients affected by unilateral sudden sensorineural hearing loss (SSHL) and uni-/bilateral definite MD, who had undergone MR imaging at our department from July 2016 to January 2018. According to the American Academy of Otolaryngology-Head and Neck Surgery (AAO-HNS) guidelines, SSHL was defined by a sensorineural hearing loss of at least 30 decibels over 3 contiguous frequencies occurring within a 72-hour period. ${ }^{8,9}$ The diagnosis of definite MD fulfilled the criteria of both the Bárány Society and the AAO-HNS. According to the Bárány Society guidelines, definite $\mathrm{MD}$ was diagnosed if there was a history of at least 2 definitive spontaneous episodes of vertigo lasting 20 minutes or longer, tinnitus, or aural fullness and audiometrically documented sensorineural hearing loss of at least $30 \mathrm{~dB}$ (or low-to-medium frequency sensorineural hearing loss). ${ }^{3}$ According to the AAO-HNS, definite MD was diagnosed if there was a history of at least two spontaneous episodes of vertigo lasting 20 minutes to 12 hours; audiometrically documented low-to-medium frequency senso- rineural hearing loss in 1 ear, defining the affected ear on at least 1 occasion before, during, or after one of the episodes of vertigo; and fluctuating aural symptoms (hearing, tinnitus, or fullness) in the affected ear, not better accounted for by another vestibular diagnosis. ${ }^{10}$

Exclusion criteria were the following: a history of neurologic or psychiatric disorders; systemic autoimmune diseases; previous otosurgical procedures; middle ear pathologies; and other causes of sensorineural hearing loss, such as inherited hearing loss, the use of ototoxic drugs, noise trauma, exposure to industrial solvents, labyrinthine fistula, and/or previous episodes of SSHL.

\section{Audiologic Assessment}

Diagnoses of SSHL and definite MD were made by an otolaryngologist by otomicroscopy, vestibular evaluation, complete audiologic examination by pure-tone audiometry with measurement of air conduction at all octave frequencies between 125 and $8000 \mathrm{~Hz}$ and of bone conduction thresholds at $250-4000 \mathrm{~Hz}$, immittance audiometry, speech audiometry, and distortion product otoacoustic emissions. The functional level score of MD was measured on the day of MR imaging for each patient. ${ }^{10}$ In patients with unilateral SSHL and unilateral $\mathrm{MD}$, the unaffected contralateral ear was defined as the healthy control (HC) and normal in a patient with MD (nMD), respectively, if the audiologic assessment revealed normal hearing, including the presence of distortion product otoacoustic emissions. Thus, after the audiologic assessment, ears could be categorized to 4 types: $\mathrm{HC}, \mathrm{SSHL}, \mathrm{MD}$, and $\mathrm{nMD}$.

\section{MR Imaging Acquisition and Image Analysis}

All patients were imaged on a 3T Achieva scanner (Philips Healthcare, Best, the Netherlands) using a 32-channel head coil. The imaging protocol consisted of sequences performed for wholebrain evaluation (axial diffusion-weighted sequence and 3D FLAIR) and specific sequences for temporal bone assessment (Table 1). Contrast-agent (gadoteridol, ProHance; Bracco Diagnostics, Princeton, New Jersey) was administered intravenously at the recommended dose $(0.2 \mathrm{mmol} / \mathrm{kg})$. MR imaging acquisition data are listed in Table 1. According to our protocol, all patients with SSHL were scanned within 10 days after onset.

Two observers, a senior neuroradiologist and a radiology resident with 6 years and 6 months of experience in otoradiology, respectively, assessed the delayed 3D-FLAIR images independently and, in a second reading session, in consensus. Multiplanar reconstructions were obtained using the local PACS viewer with a slice thickness of $0.33 \mathrm{~mm}$. Image analysis was conducted using 2 oblique planes: parasagittal, parallel to the superior semicircular canal; para-axial, parallel to the lateral semicircular canal (Fig 1). According to our new proposed method, readers had to report when the VES bulged in the third inferior portion of the vestibule, which meant below the para-axial plane at the level of the lateral 


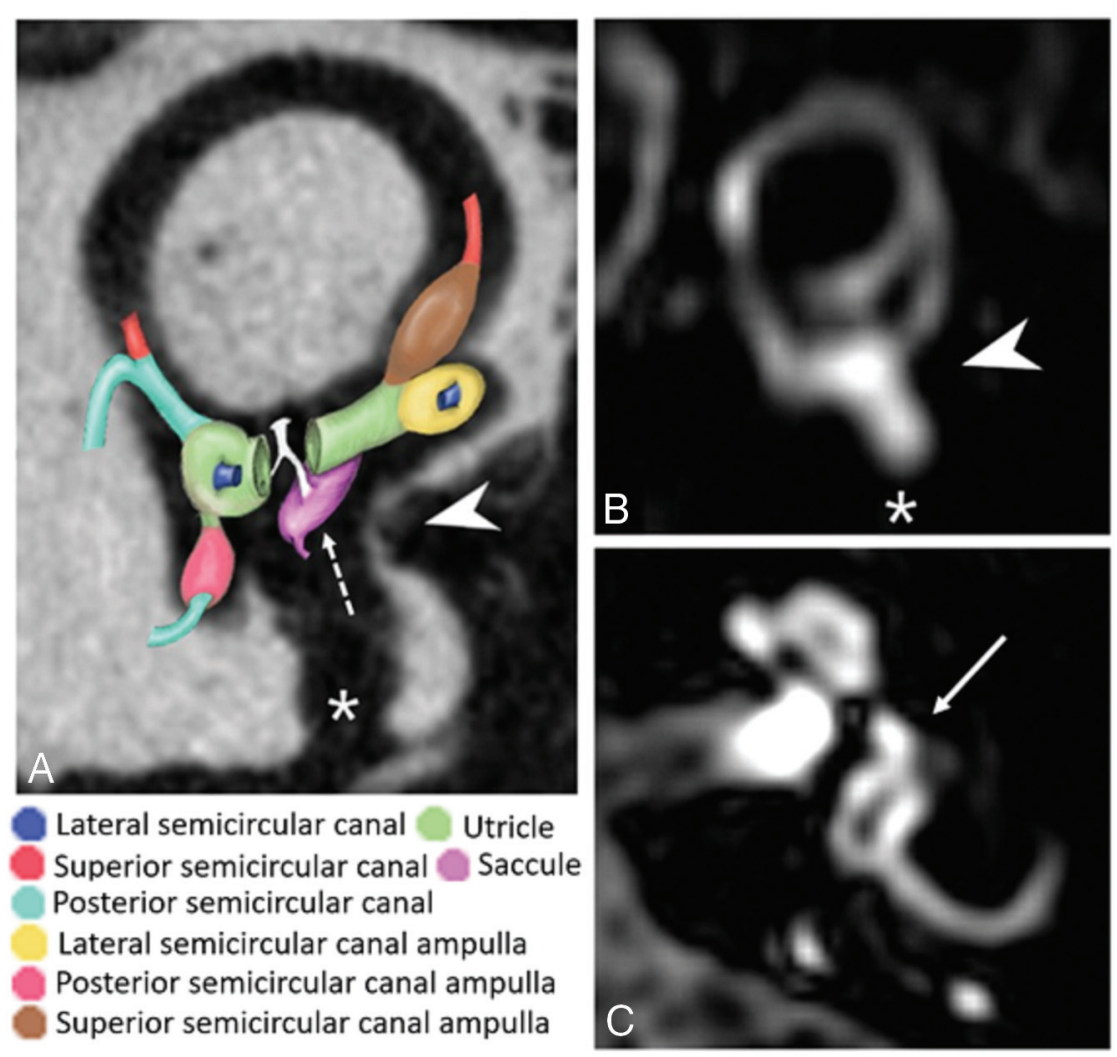

FIG 1. Left ear, $A$, Flat panel CT scan (isotropic voxel, $0.15-\mathrm{mm}$ resolution) of a cadaver temporal bone specimen with a superimposed 3D colored schematic representation of the normal VES on the oblique sagittal plane parallel to the superior semicircular canal. On this plane, the normal saccule (dotted arrow) is more medially and posteriorly located compared with the utricle. The utricle does not protrude into the inferior portion of the vestibule, and the VES does not contact the round (asterisk) and oval (arrowhead) windows. B, MR imaging oblique sagittal reconstruction parallel to the superior semicircular canal of a healthy ear shows superiorly the VES and inferiorly the perilymph filling the inferior third of the vestibule with preservation of the perilymph signal medial to the oval window (arrowhead) and round window (asterisk). C, MR imaging axial reconstruction parallel to the lateral semicircular canal at the inferior third of the vestibule in a healthy subject, showing the vestibule filled by the perilymph (arrow).

semicircular canal (Fig 2), and contacted the oval window as indicated by the disappearance of the gadolinium-enhanced perilymph behind the oval window on the parasagittal plane at the level of the superior semicircular canal. This sign was defined as the vestibular endolymphatic space contacting the oval window (VESCO).

In addition, only in the in-consensus reading section, the readers assessed the presence of cochlear hydrops $(\mathrm{CH})$ and $\mathrm{VEH}$ according to the classic criteria by Nakashima et al. ${ }^{5} \mathrm{VEH}$ was assessed by calculating the ratio of the area of the VES to that of the vestibular space. VEH was defined as "absent" if the ratio was $<33.3 \%$; "mild," from $33.3 \%$ to $50 \%$; and "significant," if $>50 \%$. $\mathrm{CH}$ was defined as "absent" if no displacement of the Reissner membrane was detected; "mild" if a displacement of the Reissner membrane was detected but the area of the cochlear endolymphatic space did not exceed the area of the scala vestibuli; and "significant" if the area of the endolymphatic space exceeded the area of the scala vestibuli.

The presence or absence of BLB breakdown of the inner ear structures was also assessed in patients with MD. The BLB breakdown was defined as a higher perilymph gadolinium-enhancement of the inner ear structures compared with the contralateral ones (Fig 3). If the enhancement was symmetric in a subject, BLB breakdown was judged absent.

\section{Statistical Analysis}

The interobserver agreement was calculated with the Cohen $\kappa$ coefficient. The clinical examination was used as a reference standard. Because we performed a per-ear analysis, we calculated the intraclass correlation coefficient to demonstrate the independence of measurements within a subject. Using the in-consensus reading for VESCO, the Nakashima method, and both combined, we calculated the sensitivity, specificity, positive predictive value (PPV), and negative predictive value (NPV) in differentiating MD ears from other ears (HC, nMD, SSHL). The McNemar test was used to compare the sensitivity and specificity between VESCO alone and VESCO combined with $\mathrm{CH}$ according to the Nakashima method. We used the Mann-Whitney $U$ test to investigate the association between BLB breakdown and the functional level score in patients with MD. Statistical analysis was performed using SPSS, Version 20.0 (IBM, Armonk, New York). Significance was defined at $P<.05$.

\section{RESULTS}

We included 24 patients affected by unilateral SSHL (male/female: 11:13; mean age, $54.9 \pm 11.7$ years) and 25 patients affected by definite MD (male/female: 15:10; mean age, $60.6 \pm 18.6$ years). There were no significant differences in the mean ages $(P=.08)$ or sex $(P=.48)$ of the SSHL and MD groups. Twenty-three of 25 patients with MD had unilateral disease (10 right, 13 left), and 2 had bilateral disease. The mean interval of disease duration in the patients with MD was 6.4 years (range, 1-25 years). No MD ears showed an air-bone gap at the audiometric examination. The functional level score at the time of MR imaging was 1 in 7 patients, 2 in 6 patients, 3 in 4 patients, 4 in 3 patients, 5 in 6 patients, and 6 in 1 patient.

We therefore analyzed 98 ears: 27 with MD, 24 with SSHL, 24 $\mathrm{HC}$, and 23 nMDs. VESCO was detected in 22 of $27 \mathrm{MD}$ ears (true-positives), 1 of 24 SSHL ears, 1 of $24 \mathrm{HC}$ ears, and 1 of 23 nMD ears (false-positives). The intraclass correlation coefficient of within-subject ear measurements was 0.07 with a $95 \% \mathrm{CI}$, $-0.20-0.35$ [null-hypothesis intraclass correlation coefficient $=$ $0 ; F(48,48)=1.16, P=.30]$. Data on the sensitivity, specificity, $\mathrm{PPV}$, and NPV in differentiating MD ears from other ears are shown in Table 2. The interreader agreement was almost perfect (Cohen $\kappa=0.867$; 95\% CI, 0.689-1).

According to the Nakashima score, VEH (32 mild, 10 severe) 


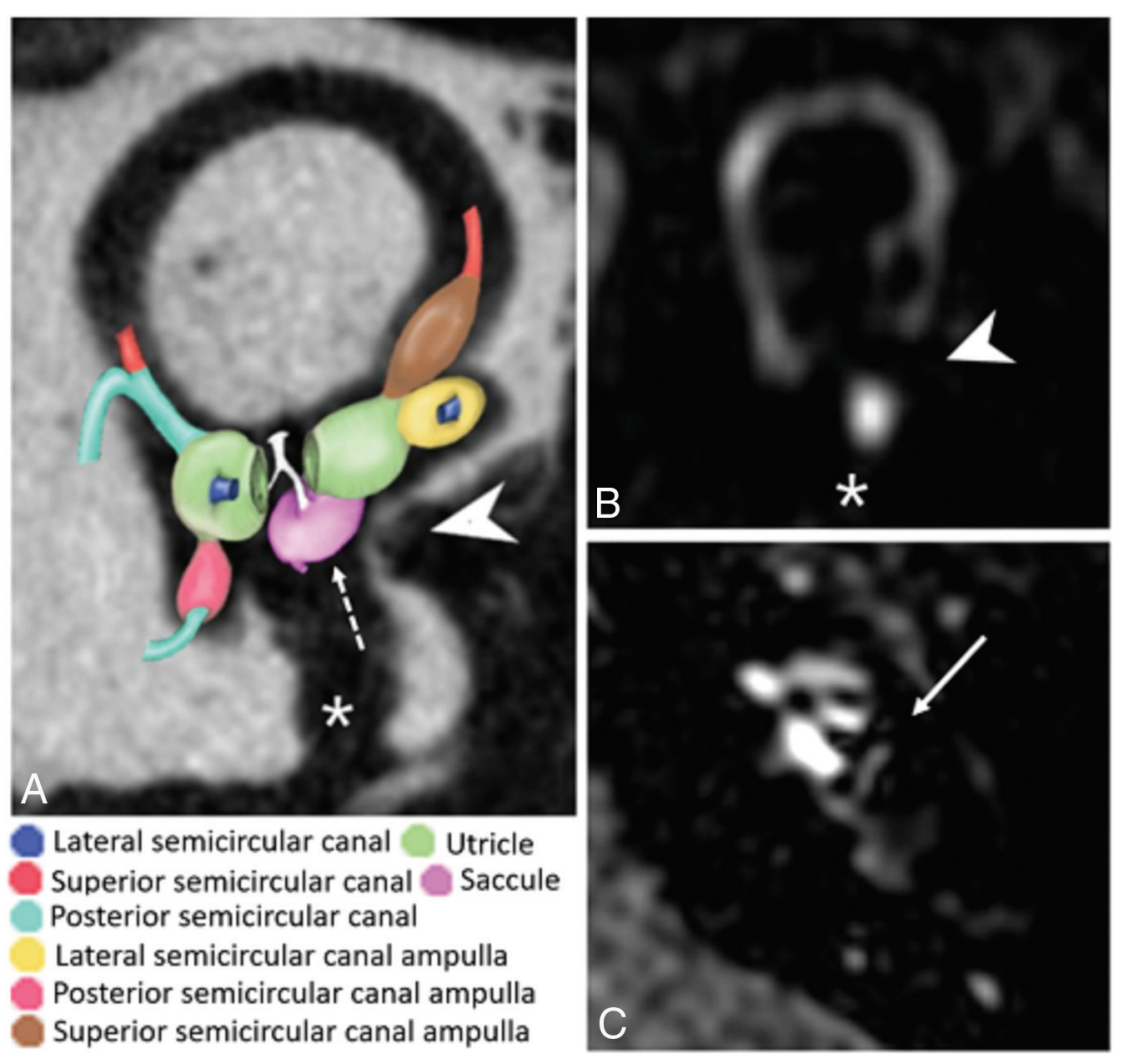

FIG 2. Left ear. A, Flat panel CT scan (isotropic voxel, $0.15-\mathrm{mm}$ resolution) of a cadaver temporal bone specimen with superimposed 3D colored schematic representation of the VES on the oblique sagittal plane parallel to the superior semicircular canal, as suggested in patients with MD. The utricle bulges into the inferior third of the vestibule, and the saccule (dotted arrow) bulges more medially; thus, the VES contacts the oval window (arrowhead). The asterisk indicates the round window and the dotted arrow indicates the saccule. $B, M R$ imaging oblique sagittal reconstruction parallel to the superior semicircular canal of an MD ear shows enlargement of the VES bulging into the inferior third of the vestibule and contacting the oval window (arrowhead), with the consequent absence of the normal perilymph signal behind the stapes footplate (asterisk indicates the round window). C, MR imaging axial reconstruction parallel to the lateral semicircular canal at the inferior third of the vestibule in a patient with MD shows the VES contacting the oval window (arrow indicates enlargement of the VES bulging into the inferior third of the vestibule and contacting the oval window).

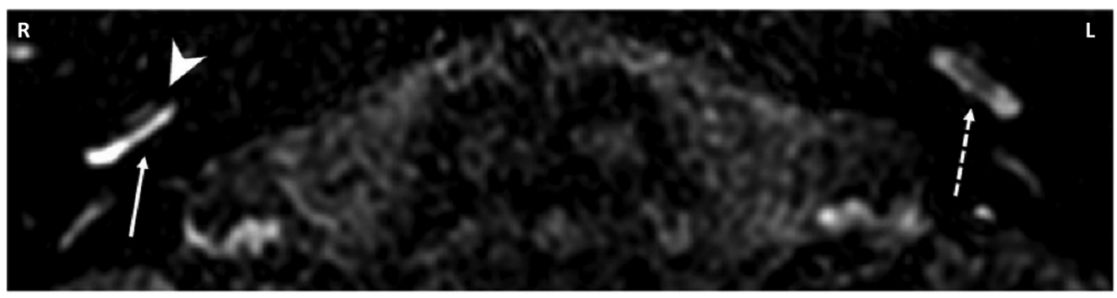

FIG 3. Four-hour-delayed postcontrast 3D-FLAIR MR axial image through the basal turns of the cochleae. The right (R) MD ear shows cochlear hydrops (arrowhead) and marked contrast enhancement (arrow) compared with the contralateral (L indicates left) healthy ear (dotted arrow), suggesting BLB breakdown.

Table 2: Contact between the oval window and the saccule in sagittal oblique plane (VESCO)

\begin{tabular}{lcccc}
\hline & $\begin{array}{c}\text { SE (\%) } \\
(95 \% \mathrm{CI})\end{array}$ & $\begin{array}{c}\text { SP (\%) } \\
(95 \% \mathrm{CI})\end{array}$ & $\begin{array}{c}\text { PPV (\%) } \\
(95 \% \mathrm{CI})\end{array}$ & $\begin{array}{c}\text { NPV (\%) } \\
(95 \% \mathrm{CI})\end{array}$ \\
\hline $\begin{array}{l}\text { Symptomatic MD vs } \\
\text { asymptomatic MD }\end{array}$ & $81(61-93)$ & $96(76-100)$ & $96(76-100)$ & $81(61-93)$ \\
$\begin{array}{l}\text { Symptomatic MD vs healthy } \\
\text { Symptomatic MD vs SSHL }\end{array}$ & $81(61-93)$ & $96(77-100)$ & $96(76-100)$ & $82(62-93)$ \\
Symptomatic MD vs other & $81(61-93)$ & $96(77-100)$ & $96(76-100)$ & $82(62-93)$ \\
\hline
\end{tabular}

Note:-SE indicates sensitivity; SP, specificity; PPV, positive predictive value; NPV, negative predictive value. was correctly detected in 20 of $27 \mathrm{MD}$ ears (true-positives) and misdiagnosed (in all cases as mild hydrops) in 10 SSHL ears, $4 \mathrm{HC}$ ears, and $8 \mathrm{nMD}$ ears (falsepositives). Furthermore, $\mathrm{CH}$ was correctly detected in 20 of $27 \mathrm{MD}$ ears (truepositives) and misdiagnosed in 4 SSHL ears, $1 \mathrm{HC}$ ear, and $5 \mathrm{nMD}$ ears (falsepositives). Data on the sensitivity, specificity, PPV, and NPV in differentiating MD ears from other ears are shown in Tables 3 and 4 .

In Table 5, the comparison between VESCO and VEH and $\mathrm{CH}$ according to the Nakashima criteria is reported in patients with MD. VESCO or $\mathrm{CH}$ or both were detected in 24 of $27 \mathrm{MD}$ ears and in 11 of 71 other ears (HC, SSHL, nMD). Thus, the presence of at least 1 of these imaging biomarkers did not show a significant difference in terms of sensitivity (89\%; 95\% CI, 70\%-97\%; McNemar test, $P=.50)$ compared with VESCO alone, but it caused a significant reduction of the specificity ( $85 \% ; 95 \% \mathrm{CI}$, $74 \%-92 \%$; McNemar test, $P=.008)$ in differentiating MD ears from the others.

BLB breakdown was identified in 14 of 23 patients with unilateral MD, always on the side of the affected ear, and in 1 of 2 patients with bilateral MD. The functional level score at the time of the MR imaging study was significantly higher in patients with BLB breakdown $(P=.03)$.

\section{DISCUSSION}

Our study showed that VESCO alone has high specificity (96\%) and PPV $(88 \%)$ in differentiating MD ears from other ears. On the contrary, the combined evaluation of VESCO and $\mathrm{CH}$ according to the Nakashima method resulted in a significant reduction of the specificity ( $85 \%$; McNemar test, $P=$ .008 ), without a significant advantage in terms of sensitivity (89\%; McNemar test, $P=.50)$. These results suggest that VESCO could be used to confirm MD after clinical examination. However, the good but not excellent sensitivity of VESCO $(81 \%)$ prevents radiologists from using it as biomarker to definitively rule out MD.

Most studies published on MR imaging of MD used the Nakashima score to identify $\mathrm{VEH}$ and $\mathrm{CH}$, but this method showed low specificity in the diagnosis 
Table 3: Vestibular endolymphatic hydrops according to Nakashima grading

\begin{tabular}{lcccc}
\hline & $\begin{array}{c}\text { SE (\%) } \\
(95 \% \mathrm{Cl})\end{array}$ & $\begin{array}{c}\text { SP (\%) } \\
(95 \% \mathrm{Cl})\end{array}$ & $\begin{array}{c}\text { PPV (\%) } \\
(95 \% \mathrm{CI})\end{array}$ & $\begin{array}{c}\text { NPV (\%) } \\
(95 \% \mathrm{Cl})\end{array}$ \\
\hline $\begin{array}{l}\text { Symptomatic MD vs } \\
\text { asymptomatic MD }\end{array}$ & $74(53-88)$ & $65(43-83)$ & $71(51-86)$ & $68(45-85)$ \\
$\begin{array}{l}\text { Symptomatic MD vs healthy } \\
\text { Symptomatic MD vs SSHL }\end{array}$ & $74(53-88)$ & $83(62-95)$ & $83(62-95)$ & $74(53-88)$ \\
Symptomatic MD vs other & $74(53-88)$ & $58(37-77)$ & $67(47-82)$ & $67(43-85)$ \\
\hline
\end{tabular}

Note:-SE indicates sensitivity; SP, specificity.

\begin{tabular}{lcccc}
\multicolumn{5}{l}{ Table 4: Cochlear hydrops according to Nakashima grading } \\
\hline & $\begin{array}{c}\text { SE (\%) } \\
(95 \% \mathrm{Cl})\end{array}$ & $\begin{array}{c}\text { SP (\%) } \\
(95 \% \mathrm{Cl})\end{array}$ & $\begin{array}{c}\text { PPV (\%) } \\
(95 \% \mathrm{CI})\end{array}$ & $\begin{array}{c}\text { NPV (\%) } \\
(95 \% \mathrm{CI})\end{array}$ \\
\hline $\begin{array}{l}\text { Symptomatic MD vs } \\
\text { asymptomatic MD }\end{array}$ & $74(53-88)$ & $78(56-92)$ & $80(59-92)$ & $72(50-87)$ \\
Symptomatic MD vs healthy & $74(53-88)$ & $96(77-100)$ & $95(74-100)$ & $77(57-89)$ \\
Symptomatic MD vs SSHL & $74(53-88)$ & $83(62-95)$ & $83(62-95)$ & $74(53-88)$ \\
Symptomatic MD vs other & $74(53-88)$ & $86(75-93)$ & $67(47-82)$ & $90(79-95)$ \\
\hline
\end{tabular}

Note:-SE indicates sensitivity; SP, specificity.

Table 5: Presence of VESCO versus VEH and $\mathrm{CH}$ according to the Nakashima criteria in patients with MD

\begin{tabular}{lcccccc}
\hline & \multicolumn{3}{c}{ VEH } & & \multicolumn{2}{c}{ CH } \\
\cline { 2 - 4 } \cline { 7 - 8 } \multicolumn{1}{c}{ VESCO } & No & Mild & Severe & & No & Yes \\
$(n=7)$ & $(n=11)$ & $(n=9)$ & & $(n=7)$ & $(n=20)$ \\
\hline No $(n=5)$ & $2(40 \%)$ & $3(60 \%)$ & $0(0 \%)$ & & $3(60 \%)$ & $2(40 \%)$ \\
Yes $(n=22)$ & $5(23 \%)$ & $8(36 \%)$ & $9(41 \%)$ & & $4(18 \%)$ & $18(82 \%)$ \\
\hline
\end{tabular}

of $\mathrm{MD}$, resulting in poor utility in clinical practice. The main limitations of this method are the following: 1) the conspicuity of the endolymphatic space depends on the MR image parameters such as TI, making results from different imaging centers less comparable; and 2) the endolymphatic space is a volume, with extreme variability in morphology ${ }^{11}$; therefore, the evaluation of its expansion simply on a single axial plane is not a suitable method to obtain a reliable estimate of the hydrops state.

However, MR imaging evidence from the literature using the Nakashima score is in line with the pathologic findings that have been described in cadavers, suggesting that $\mathrm{CH}$ and $\mathrm{VEH}$ are not exclusive findings in patients with MD and are probably not always pathologic. Thus, the ambitious objective for radiologists is to determine a method to distinguish clinically relevant hydrops. We support the idea that morphology-based imaging methods, such as ours, can be more accurate than quantitative-based ones, such as the Nakashima score, to reach this goal. Attyé et $\mathrm{al}^{6}$ pursued this objective using a saccule-to-utricle ratio of $\geq 1$ as a biomarker for MD, reaching low sensitivity (50\%) but high specificity $(100 \%)$ in differentiating the symptomatic ears of patients with MD from the asymptomatic ears of healthy volunteers. However, it remains unclear what the diagnostic role of the saccule-toutricle ratio is in differentiating MD ears from ears affected by other otologic disorders. We find the saccule-to-utricle ratio difficult to apply in clinical practice for the following reasons: 1) When there is $\mathrm{VEH}$, there is the effacement of the perilymph between the saccule and the utricle, thus making it impossible to delineate the boundaries of these structures; and 2) Attyé et al calculated the saccule-to-utricle ratio in a parasagittal plane along the major axis of the vestibule, but the saccule and the utricle are not visible together in this plane because the saccule is more medially located.
Attyé et $\mathrm{al}^{6}$ reported the contact of the VES with the oval window in 9/30 patients with $\mathrm{MD}$, all with the sacculeto-utricle ratio. Our data disagree with this observation. The discrepancy could be due to technical differences in the imaging analysis. Attyé et al did not clearly state their method of defining the contact between the saccule and the stapes footplate, which is surely different from ours: We used a parasagittal plane parallel to the lateral semicircular canal, which does not correspond to the parasagittal plane along the long axial axis of the vestibule as represented by these authors. Recently, some authors reported that patients with significant VEH adjacent to the stapes footplate showed higher average air-bone gaps at $250 \mathrm{~Hz}$ compared with patients with nonadjacent significant $\mathrm{VEH}$.

In contrast, we did not find air-bone gaps in our patients with MD. ${ }^{12}$ First, audiograms of patients with MD showed unexplained air-bone gaps at low frequencies from 13\% to $32 \%$ in the literature, even though no middle ear pathology could be demonstrated. ${ }^{13,14}$ In addition, some methodologic differences between our study and the aforementioned study ${ }^{12}$ should be stated to understand why the results are in disagreement. The authors of this study enrolled not only patients with MD ( $66 \%$ of the total) but also patients with other otologic disorders who presented with severe hydrops according to the Nakashima score. As evidenced by our results, severe hydrops was found only in 9/22 (41\%) patients with MD with VESCO. According to these observations, the populations of the studies are not comparable. In addition, the authors used "HYDROPS" imaging, performed by subtracting the positive endolymph images from the positive perilymph images. This type of postprocessing transformation can alter the MR imaging anatomy of the endolymphatic space by inducing researchers to incorrectly locate the endolymph liquid within the inner ear structures for problems of registration between the 2 MR images. This limitation could be more relevant when VEH should be defined as "nonadjacent" or "adjacent" according to the presence or absence of a black area of endolymph underneath the stapes footplate.

Our study showed that patients with MD with BLB breakdown have a higher functional level at the time of the MR imaging than those without BLB breakdown. Similar to our results, in a recent MR imaging study, it was noted that the permeability of the capillaries in the inner ear of patients with MD was significantly greater than that in the inner ear of patients with SSHL. ${ }^{15}$ These findings are in agreement with the hypothesis that MD may be caused by dysfunction in the BLB as confirmed by the pathologic changes that were described in the capillary lumens of MD cadavers. ${ }^{16}$ The finding of increased vesicular transport in the endothelial cells of the capillaries, vascular endothelial cell degenerative changes, and the thickening of the basement membrane of the capillaries in Menière disease raises the possibility of an inflam- 
matory pathology similar to that proposed as causative in autoimmune inner ear disease. ${ }^{17}$

Some limitations of our study should be acknowledged. Our standard reference for the definition of MD was clinical instead of histopathologic. Furthermore, while enrolling only patients with definite MD, we did not test the accuracy of VESCO in those clinical conditions, such as possible or probable MD or MD-mimicking disorders, which represent the true diagnostic challenges in clinical practice. Thus, the reported specificity of VESCO could be overestimated. For example, is VESCO accurate in early differentiate recurrent peripheral vestibulopathy from MD? Future studies should therefore aim to answer this question using clinical follow-up or histology as a reference standard. Because in SSHL we performed MR imaging within 10 days after the onset of the pathology, we cannot exclude the possibility that these patients could develop late vestibular hydrops and that VESCO could be detected in them later.

We did not correlate imaging results with audiometric tests, the duration of MD, or vestibular-evoked myogenic potentials. In particular, the vestibular-evoked myogenic potentials are a reliable tool for the investigation of saccular function ${ }^{18}$; thus, they can elucidate the role of the saccule in the definition of VESCO, considering that in our opinion, it is impossible to clearly delineate the saccule from the other endolymphatic structures when marked hydrops is present. Some technical aspects in the imaging acquisition could have influenced our results; a recent study has shown that it is easy to shift from the normal condition to VEH disease simply by varying the TI sequence by $100 \mathrm{~ms} .{ }^{19}$ The same group of researchers suggested that different contrast agents can provide different anatomic details regarding endolymphatic space and the semicircular canals. ${ }^{19}$

\section{CONCLUSIONS}

VESCO is a promising MR imaging biomarker for MD and BLB breakdown, as depicted by the enhancement on MR imaging, to identify patients with higher functional levels probably due to florid inflammatory activity within the inner ear structures.

Disclosures: Diego Zanetti-UNRELATED: Payment for Lectures Including Service on Speakers Bureaus: Advanced Bionics, Staefa (Switzerland), Comments: honorarium and travel expenses for lecturing at an international course on cochlear implants, Hannover, Germany 2017.

\section{REFERENCES}

1. Gürkov R. Menière and friends: imaging and classification of hydropic ear disease. Otol Neurotol 2017;38:e539-44 CrossRef Medline

2. Merchant SN, Rauch SD, Nadol JB Jr. Meniere's disease. Eur Arch Otorhinolaryngol 1995;252:63-75 Medline

3. Lopez-Escameza JA, Careyb J, Chung WH, et al. Diagnostic criteria for Menière's disease: consensus document of the Bárány Society, the Japan Society for Equilibrium Research, the European Academy of Otology and Neurotology (EAONO), the American Academy of Otolaryngology-Head and Neck Surgery (AAO-HNS) and the Korean Balance Society [in Spanish]. Acta Otorrinolaringol Esp 2016;67: 1-7 CrossRef Medline

4. Sepahdari AR, Ishiyama G, Vorasubin N, et al. Delayed intravenous contrast-enhanced 3D FLAIR MRI in Meniere's disease: correlation of quantitative measures of endolymphatic hydrops with hearing. Clin Imaging 2015;39:26-31 CrossRef Medline

5. Nakashima T, Naganawa S, Pyykko I, et al. Grading of endolymphatic hydrops using magnetic resonance imaging. Acta Otolaryngol Suppl 2009:5-8 CrossRef Medline

6. Attyé A, Eliezer M, Boudiaf N, et al. MRI of endolymphatic hydrops in patients with Meniere's disease: a case-controlled study with a simplified classification based on saccular morphology. Eur Radiol 2017;27:3138-46 CrossRef Medline

7. Conte G, Lo Russo FM, Calloni SF, et al. MR imaging of endolymphatic hydrops in Menière's disease: not all that glitters is gold. Acta Otorhinolaryngol Ital 2018;38:369-76.

8. Stachler RJ, Chandrasekhar SS, Archer SM, et al; American Academy of Otolaryngology-Head and Neck Surgery. Clinical practice guideline: sudden hearing loss. Otolaryngol Head Neck Surg 2012;146(3 Suppl): S1-35 CrossRef Medline

9. Rauch SD. Clinical practice: idiopathic sudden sensorineural hearing loss. N Eng J Med 2008;359:833-40 CrossRef Medline

10. Committee on Hearing and Equilibrium guidelines for the diagnosis and evaluation of therapy in Menière's disease: American Academy of Otolaryngology-Head and Neck Foundation, Inc. Otolaryngol Head Neck Surg 1995;113:181-85 CrossRef Medline

11. Pender DJ. Membrane stress in the human labyrinth and Meniere disease: a model analysis. Int Arch Otorhinolaryngol 2015;19:336-42 CrossRef Medline

12. Sugimoto $S$, Yoshida $T$, Teranishi M, et al. The relationship between endolymphatic hydrops in the vestibule and low-frequency airbone gaps. Laryngoscope 2018;128:1658-62 CrossRef Medline

13. Lee HJ, Jeon JH, Park S, et al. Prevalence and clinical significance of spontaneous low-frequency air-bone gaps in Menière's disease. Otol Neurotol 2014;35:489-94 CrossRef Medline

14. Muchnik C, Hildesheimer M, Rubinstein M, et al. Low frequency air-bone gap in Menière's disease without middle ear pathology: a preliminary report. Am J Otol 1989;10:1-4 CrossRef Medline

15. Pakdaman MN, Ishiyama G, Ishiyama A, et al. Blood-labyrinth barrier permeability in Menière's disease and idiopathic sudden sensorineural hearing loss: findings on delayed postcontrast 3D-FLAIR MRI. AJNR Am J Neuroradiol 2016;37:1903-08 CrossRef Medline

16. Ishiyama $G$, Lopez IA, Ishiyama $P$, et al. The blood labyrinthine barrier in the human normal and Meniere's disease macula utricle. $S c i$ Rep 2017;7:253 21 CrossRef Medline

17. Neng L, Zhang F, Kachelmeier A, et al. Endothelial cell, pericyte, and perivascular resident macrophage-type melanocyte interactions regulate cochlear intrastrial fluid-blood barrier permeability. $J$ Assoc Res Otolaryngol 2013;14:175-85 CrossRef Medline

18. de Waele C, Tran Ba Huy P, Diard JP, et al. Saccular dysfunction in Menière's patients: a vestibular-evoked myogenic potential study. Ann N Y Acad Sci 1999;871:392-97 CrossRef

19. Eliezer M, Gillibert A, Tropres I, et al. Influence of inversion time on endolymphatic hydrops evaluation in 3D-FLAIR imaging. J Neuroradiol 2017;44:339-43 CrossRef Medline 